\title{
LOS PROCESOS DE FAMILIA DESDE LA ÓPTICA DEL ACCESO A LA JUSTICIA: HACIA LA CONSOLIDACIÓN DEL DERECHO PROCESAL DE FAMILIA
}

\author{
Marco Antonio Celis VásqueZ*
}

\section{Resumen}

El Derecho Procesal de Familia se ha consolidado en la última década, al reconocérsele doctrinariamente características y peculiaridades propias y la existencia de principios autónomos, así como la exigencia de contar con un juez capacitado de manera especial en este tipo de procesos, con una formación técnico - jurídica, en asuntos de índole familiar y una visión, completamente distinta a los demás procesos civiles. Es importante recalcar la visión que con respecto al juez posee el doctor Rogelio Llerena Quevedo, quien señala que: "el juez interprete y administrador ecuánime de la justicia, solo puede ser comprendido como uno entre los mejores seres humanos. Esto implica que aún siendo el mejor, sigue siendo humano y, por lo tanto susceptible de error. Por tales razones, debe siempre tener como guía un Código de ética que sirva de guía para un buen juez" (Llerena Quevedo, Rogelio).

Palabras Clave: Derecho procesal de familia - Interés superior del niño - Modelo de interpretación dinámica - Catálogo mínimo de derechos Convención sobre los derechos del niño - Objetividad - Estado de justicia.

\begin{abstract}
Family Procedural Law has been consolidated in the last decade as scholar doctrinary opinions have acknowledged its own characteristics and peculiarities, and the existence of self-governing principles, as well the requirement to have a judge specialized in this kind of proceedings, with a legal-technical training in family matters, and with a perspective different from the other civil actions. It is important to point out Rogelio Llerena Quevedo's view as to the role of a judge. He states that: "an unbiased judge interpreting and administering justice could only be taken as one of the best human being. This implies that even if he is the best, he is still human, and therefore, capable of making mistakes. Based on those reasons, there should always be a Code of Ethics as a guide for a good judge".
\end{abstract}

Key words: Family Procedural Law - Superior interest of children - Dynamic interpretation model - Minimum rights table - Convention on the Rights of the Child - Objectivity - Rule of justice.

\section{Sumario}

1. Introducción. 2. Características peculiares de los procesos de familia. a) Peculiaridades de los litigios. b) Audiencias especiales. c)Presencia necesaria de órganos de apoyo. d) Integración multidisciplinaria. e) Una permeable tarea hermenéutica finalista y previsora de lo que eventualmente pueda suceder.

\footnotetext{
* Juez Especializado de Familia de la Corte Superior de Justicia de La Libertad - Poder Judicial de Perú.
} 
f) La presencia de la prueba científica. 3. Principios del proceso de familia.

a) Gratuidad y acceso a la justicia. b) Publicidad de los procedimientos. c) Inmediación, oralidad, privacidad y acentuación de la función conciliadora. d) Aplicación del principio del favor probationis. e) Oponibilidad erga omnes de la sentencia. f) Cooperación interdisciplinaria. g) Simplificación de los proceso cautelares. h) Principio de la perpetuatio jurisidictionis. i) El interés superior del niño. 4. Consideraciones procesales sobre derecho de familia. a) El modelo de interpretación estática. b) El modelo de interpretación dinámica. 5. Consideraciones procesales en materia de derecho de familia aplicando el modelo de interpretación dinámica. 6. El interés superior del niño en la interpretación del Tribunal Constitucional. a) El interés superior del niño y el derecho a vivir en familia; b) El interés superior del niño y el derecho a la educación; y, c) El interés superior del niño y el derecho a la libertad personal. 7. Determinación del interés superior del niño para el Tribunal Constitucional. 8. El interés superior del niño en el proceso civil, tutelar y de familia. 9. Conclusiones.

\section{INTRODUCCIÓN}

Los años setenta, tanto España como Italia mostraron la apertura a las interesantes transformaciones de los procesos laborales y de familia, pues se empezó a privilegiar el debate y el contradictorio, privilegiando el principio de inmediación, efectivizando la bilateralidad e igualdad de trato. Desde tal perspectiva configuran las garantías nacionales e internacionales del proceso justo.

"El compromiso del derecho procesal moderno es con la persona, con el hombre consumidor de la justicia, el incapaz y el desprotegido. En la dimensión de estas controversias todas ellas se visten con una llamativa luminosidad que permite registrar a los procesos familiares en lo avanzado del renovado derecho procesal que va tomando cabal posición al confirmar el tránsito manifiesto del Estado de derecho al Estado de justicia"1.

Un análisis moderno de los contenidos de los temas más frecuentes en la esfera del derecho de familia, constituye su espesor, versatilidad y transmutación.

El derecho procesal se halla ante dos retos cada vez mas sofisticados, la sociedad sumida en riesgos más agobiantes y de otra escala (internet, comunicación global, delincuencia, corrupción generalizada, pornografía, etc.), que excluye de las teorías y operadores de cualquier actitud quietista y evasiva, desoyendo el torrente de críticas y el clamor que nos insta a no demorarnos, a superar el inmovilismo de la justicia y a su mala receptividad en el seno de la comunidad, que pareciera, solo sabe hacer camino como un andar divorciado de la aceleración del contexto. 
Se viene marcando con insistencia los profundos cambios ya acaecidos en la alborada de una nueva cultura jurídica que coloca a los fenómenos jurídicos familiares en el objeto de un estudio dinámico de tales hechos familiares, los mismos que deben ser analizados, tratados con una metodología propia y pautas hermenéuticas de otra altura y en otro contexto y en muy diferente estima, por el privilegio de los valores y principios.

\section{CARACTERÍSTICAS PECULIARES DE LOS PROCESOS DE FAMILIA}

"Se puede afirmar que los procesos de índole familiar tienen especialísimas características que no albergan otros procesos y los cuales se detalla"2:

a) Peculiaridades de los litigios: que genera el uso marcado de la técnica de la conciliación y de la mediación, como etapa previa necesaria y de logros autocompositivos llamativos, pues se privilegia la sensatez y se deben apagar las pasiones.

b) Audiencias especiales: que permitan tener un contacto directo con las partes involucradas, necesitando además de conocimientos jurídicos, de conocimientos de psicología y pedagogía.

c) Presencia necesaria de órganos de apoyo: asistentes sociales, analistas del campo social, psicólogos, etc.

d) Integración multidisciplinaria: que se arropa en conocimientos de diversos orígenes que operan y reactivan de manera acumulativa, pues no es solo el derecho el que abastece la razonabilidad a la función jurisdiccional.

e) Una permeable tarea hermenéutica finalista y previsora de lo que eventualmente pueda suceder: todo ello a fin de impedir que se agudicen los conflictos anteriores, pues deben ser resueltos de manera oportuna.

f) La presencia de la prueba científica: que cada día se hace más gravitante.

\section{PRINCIPIOS DEL PROCESO DE FAMILIA}

El derecho de familia en su tramitación cuenta con principios propios y además principios comunes al derecho procesal, y que concurren para darle una particular fisonomía, no tanto por las formas que lo revisten, como por la intensidad, la extensión y la generalización con que aparecen reunidos y aplicados, todo lo cual contribuye a conformar un proceso singular que goza, además, de una incipiente autonomía científica e incluso legislativa. Además, resulta gravitante la labor de un juez especializado en temas de familia y especialmente en temas de niños y niñas, “[...] pues su intervención en torno al uso de los instrumentos lógicos, resulta de la distinción entre razonamiento decisorio y razonamiento justificativo" ${ }^{3}$. 
Según Jorge Kielmanovich, son inherentes al proceso de familia los siguientes principios:

\section{a) Gratuidad y acceso a la justicia}

Los procesos de familia por regla general están exentos del pago de tasas judiciales y otros derechos. Por otra parte y en cuanto al régimen de las costas, se afirma que una tendencia a prescindir para su imposición, del "principio de la derrota", pues la intervención del juez es para solucionar un problema de orden familiar y en donde inclusive existan involucrados menores de edad.

\section{b) Publicidad de los procedimientos}

La acentuación del carácter público de los procedimientos, con las excepciones reguladas expresamente cuando se afecten derechos a la intimidad o derecho de menores.

\section{c) Inmediación, oralidad, privacidad y acentuación de la función conciliadora}

"Los intereses comprometidos en el litigio de familia torna imperioso, conveniente y necesario, el contacto directo del juez con las personas que intervienen en el proceso, de modo de procurar y alcanzar, así un conocimiento de primera mano de los hechos debatidos y de los sujetos involucrados en el proceso, contacto que se propone en líneas generales a partir del sistema de las audiencias preliminares y conciliatorias" ${ }^{4}$. Así tenemos, por ejemplo, en los procesos de tenencia de menores, régimen de visitas, autorización para contraer matrimonio, etc.

\section{d) Aplicación del principio del favor probationis}

El principio del favor probationis de escaso desarrollo doctrinario y jurisprudencial explícito, pero de inocultable aplicación en nuestro medio, supone que en caso de objetivas dudas y especialmente de dificultades probatorias (como sucede en los procesos de familia) habrá de estarse por un criterio amplio a favor de la producción, admisión y eficacia de las pruebas, teniendo en consideración que los hechos llamados a constituirse en objeto de las mismas, normalmente ocurren en la intimidad del hogar, y en su caso, en presencia de testigos comprendidos dentro de la generales de la ley, extremo que obliga a valorar el comportamiento omisivo en tal contexto como prueba en contra. 


\section{e) Oponibilidad erga omnes de la sentencia}

Con relación a las sentencias que se refieren al Estado y capacidad de las personas se predica su eficacia erga omnes, en base a la indivisibilidad del estado de familia y orden público.

Sobre el particular, entiende Zannoni que, el problema de la oponibilidad del estado de familia no contrapone los efectos relativos de la cosa juzgada, porque el título que la sentencia constituye o modifica siendo oponible erga omnes, puede nuevamente ser impugnado por quienes no intervinieron en el proceso y a los cuales no alcanza la cosa juzgada.

\section{f) Cooperación interdisciplinaria}

El conflicto de familia normalmente involucra no solo a sus protagonistas, sino a los integrantes del grupo familiar conviviente, razón por la cual se establece la cooperación interdisciplinaria para solucionar o prevenir el agravamiento o extensión del problema, mediante la intervención de asistentes sociales, psicólogos, etc., adscritos al tribunal o juzgado o designados de oficio o a petición de parte.

\section{g) Simplificación de los procesos cautelares}

En los procesos de familia, las medidas cautelares adquieren un peculiar contorno, verificándose profundas modificaciones dado su carácter instrumental; a su proveimiento inaudita parte; a los presupuestos que hacen su admisibilidad y ejecutabilidad; a la facultad del órgano para ordenarlas de oficio; a la disponibilidad inmediata de su objeto; y, por fin, a su no sujeción normativa a términos de caducidad. Ejemplo: la asignación anticipada de alimentos, la internación preventiva de menores en algún establecimiento designado por el juez.

Conviene recordar que la concreta conceptualización de una medida como "cautelar", no es una cuestión académica dentro del procedimiento general, pues constituye una medida anticipada que sin lugar a dudas traerá beneficios para el sujeto o sujetos protegidos y que tratándose de menores, resulta de prioritaria atención.

\section{h) Principio de la perpetuatio jurisdictionis}

Por este principio deben promoverse así ante el juez que entiende o entendió el juicio de divorcio o separación personal, las cuestiones conexas o procesos cautelares e incidentales. 


\section{i) El interés superior del niño}

La Convención sobre los Derechos del Niño, sienta el principio de que en toda actuación judicial debe velarse por el interés superior del niño, fórmula que opera en causas, "tanto concernientes al derecho de familia como ajenas a dicha materia", sea que aquel intervenga en calidad de parte procesal (por ejemplo en la reclamación de filiación) o ya sea como simple tercero, más allá de que el mismo pueda verse, no obstante, alcanzado por el referido conflicto (proceso de divorcio separación, nulidad de matrimonio, etc.).

El interés superior del niño, se constituye de tal suerte en una valiosa y esencial herramienta para la resolución de los conflictos judiciales que pudiese comprometer o afectar a las personas, derechos e intereses de los menores, con una virtualidad y extensión que, a la par de encontrarse en permanente evolución, se vislumbra de una riqueza inconmensurable, sin perder de vista su incidencia en nuestro ordenamiento sustantivo.

\section{CONSIDERACIONES PROCESALES SOBRE DERECHO DE FAMILIA}

En la interpretación de asuntos de índole familiar, resulta de vital importancia, tener en cuenta los modelos de interpretación jurídica que al respecto nos informa la Casación $N^{\circ}$ 2414-2006-CALLAO (relativa a un caso sobre divorcio por causa de separación de hecho) que detalla los diferentes modelos de interpretación jurídica, pues superada satisfactoriamente la etapa del saneamiento procesal, el juez vuelca todo su conocimiento jurídico a la solución del conflicto, y en el caso concreto, la separación de hecho motiva que el juez utilice toda su apreciación razonada sobre el caso concreto, sin perder de vista los precedentes jurisprudenciales sobre casos similares, pero, además, no puede perderse de vista los modelos de interpretación jurídica, que en el caso de la separación de hecho, deberá tener siempre presente, al ser una causal muy amplia y compleja:

Los modelos de interpretación jurídica reconocidos preponderantemente son dos:

\section{a) El modelo de interpretación estática}

Según el cual el modelo de interpretación jurídica persigue indagar lo realmente querido por el legislador, esto es determinar la voluntad del legislador. 


\section{b) Modelo de interpretación dinámica}

Según el cual el objetivo de la interpretación no es la voluntad del legislador sino la voluntad de la ley ${ }^{5}$.

En un Estado democrático y social de derecho, aún la actividad hermenéutica de las normas debe sujetarse al principio de separación de poderes; en consecuencia, el juez no puede sujetarse a la voluntad del legislador, es decir, el juez no puede actuar como "la boca que pronuncia las palabras de la ley", como antiguamente se postulaba; por el contrario, está llamado a aplicar y a interpretar la norma jurídica en un contexto social determinado, en un tiempo y lugar en donde operan los factores sociales, económicos, políticos, culturales, entre otros, pues el derecho vigente regula las relaciones jurídicas emergentes en dicho contexto. Por ello considero que los magistrados, en la actividad interpretativa, debemos seguir principalmente el modelo dinámico, y en forma secundaria, el modelo estático, para efectos de conducir eficazmente, no solo a determinar la voluntad objetiva de las normas, sino además, concretar los valores, fines y principios vigentes en un sistema jurídico determinado: principalmente el sistema material de valores que reconoce y consagra la Constitución Política del Estado y de la concreción del valor justicia en el caso concreto.

\section{CONSIDERACIONES PROCESALES EN MATERIA DE DERECHO DE FAMILIA APLICANDO EL MODELO DE INTERPRETACIÓN DINÁMICA}

A continuación se enumera algunos casos en las que el juzgador debe aplicar el modelo de interpretación dinámica, a partir de las siguientes normas:

a) El artículo $88^{\circ}$ del Código de Niños y Adolescentes: los padres que no ejerzan la patria potestad tienen derecho a visitar a sus hijos, para lo cual deberán acreditar con prueba suficiente el cumplimiento o la imposibilidad del cumplimiento de la obligación alimentaria.

b) El artículo 345-A del Código Civil: para invocar el supuesto del inciso 12 del artículo $333^{\circ}$ del Código Civil, el demandante deberá acreditar que se encuentra al día en el pago de sus obligaciones alimentarias u otras que hayan sido pactadas por los cónyuges de mutuo acuerdo.

c) El artículo $137^{\circ}$ del Código de Niños y Adolescentes: el juez está facultado para fijar la pensión de alimentos dentro del mismo proceso en los casos de litigios de patria potestad, tenencia, régimen de visitas, etc.

d) El artículo $370^{\circ}$ del Código Procesal Civil: que, consagra el principio de la reformatio in peius o de prohibición de reforma en peor; por el cual si 
una parte recurre en apelación de una resolución, el superior solo puede reformar la resolución a su favor, jamás en su contra. En materia de protección de niños no resulta aplicable (Casación Nº 756-2005-Puno).

e) El artículo $84^{\circ}$ inciso c) del Código de Niños y Adolescentes: prevé que para el que no obtenga la tenencia o custodia del niño o niña, deberá señalarse un régimen de visitas. Esta norma es imperativa o facultativa. Qué sucede si en un proceso de tenencia no se demandó el régimen de visitas: Casación N 3172-2005-Lima.

f) El artículo $85^{\circ}$ del Código de Niños y Adolescentes: el señor Juez debe escuchar la opinión del niño y tener en cuenta la del adolescente.

g) Resolución Legislativa $N^{\circ} 28246$ que aprueba la "Convención Interamericana sobre Restitución Internacional de Menores", suscrita en la ciudad de Montevideo - Uruguay, el 15 de julio de 1989, de conformidad con los artículos $56^{\circ}$ y $102^{\circ}$, inciso 3, de la Constitución Política del Perú. Convención ratificada por el artículo $1^{\circ}$ del Decreto Supremo N $^{\circ}$ 058-2004-RE, publicado el 10 de septiembre de 2004. La presente Convención entró en vigencia para el Perú el 1 de abril de 2005.

En todos los casos enumerados, el Juez Especializado de Familia debe aplicar el modelo de interpretación dinámica, es decir, no aplicando restrictivamente lo descrito por el legislador y plasmado a través de dichas normas, sino por el contrario, confrontar todo su conocimiento especializado en la solución de la controversia de índole familiar, y en el caso de intervenir menores de edad, privilegiando la correcta aplicación del principio del interés superior del niño.

\section{EL INTERÉS SUPERIOR DEL NIÑO EN LA INTERPRETACIÓN DEL TRIBUNAL CONSTITUCIONAL}

El interés superior del niño, constituye un concepto jurídico indeterminado. En efecto, en el artículo $3^{\circ}$, primer párrafo, de la Convención, "el interés superior del niño", aparece tipificado, expresado legalmente como concepto jurídico indeterminado, por medio de una cláusula general con la que se le establece como principio y también como mandato o expresión concreta para supuestos de hecho específicos.

Coincidentemente, el Tribunal Constitucional ha precisado que el interés se expresa concluyentemente como valor que una cosa posee en sí misma y como la consecuencia de la inclinación colectiva hacia algo que resulta atractivo, apreciable y útil. 
En primer término, se debe partir de la idea matriz de que "el interés superior", a efectos jurídicos, está inicialmente en la protección de sus derechos fundamentales, y al individualizarlo habrá de garantizársele, a través de las opciones y decisiones que se adopten, los bienes y valores en que se encarnan esos derechos fundamentales.

“El interés superior del niño y del adolescente debe ser concebido como una exigencia para que las autoridades no solo se queden en dictar normas o políticas gubernamentales en abstracto, sino que deben velar por que la estricta observancia de ellas redunde en los niños y adolescentes, responsabilidad también aplicable a la comunidad"6.

Ante ello, dicho Tribunal ha aplicado el interés superior del niño en distintas situaciones:

\section{a) El interés superior del niño y el derecho a vivir en familia}

El Tribunal Constitucional resolvió un caso en el que, sin mencionar expresamente la norma citada, se refirió a los alcances de su contenido ${ }^{7}$.

Se trata de un proceso de hábeas corpus que siguiera doña Lady Rodríguez Pandero contra la Ministra del Ministerio de Promoción de la Mujer y Desarrollo Humano y el Jefe de la Oficina de Adopciones, con el objeto de que le sea entregada la menor L.E.P.D.L.R, de 3 años y 6 meses de edad, quien se encuentra internada en la Aldea Infantil "Virgen del Pilar", de la ciudad de Tarapoto.

Dicha menor, a los pocos días de nacida fue entregada por su madre biológica a la demandante para que la críe como su hija, la misma que la registró como tal ante la Municipalidad de Mariscal Cáceres. Después de dos años, la madre biológica reclamó a su hija y la denunció por rapto de menor. La demandante, al haber sido condenada en primera instancia en el proceso que se menciona, procedió a entregar a la madre biológica, con intervención del fiscal provincial. Sin embargo, ninguno de los padres biológicos cumplió sus obligaciones y posteriormente, entregaron a dicha niña a sus abuelos paternos, quienes tampoco asumieron responsabilidad alguna, teniéndola en completo estado de abandono.

Ante tales hechos, el Tribunal Constitucional aprecia la demostración de solidaridad de la demandante para con una recién nacida, en delicado estado de salud y abandonada por su madre biológica, mientras que la demandante le brindó amor, cuidados y protección. 
Al respecto el Tribunal, teniendo en consideración que la Constitución establece que la persona humana es el fin supremo de la sociedad y del Estado, y que tanto la comunidad como el Estado protegen especialmente al niño en situación de abandono; y asimismo, que el Código de los Niños y Adolescentes prescribe que en toda medida que adopte el Estado concerniente al niño, se considerará el interés superior de este y el respeto a sus derechos, y que todo menor tiene derecho a vivir, crecer y desarrollarse en el seno de una familia, declarando, por ende, fundada la demanda y ordenó que la niña debe ser entregada a la demandante.

Se advierte que, el Tribunal ha concebido al interés superior del niño como un interés autónomo, como el único relevante en este contexto social y normativo, de forma que debe sobreponerse a cualquier otro.

\section{b) El interés superior del niño y el derecho a la educación}

Aquí los problemas se han presentado en los casos en que los directores de los centros educativos (estatales y particulares), han impedido (negando o cancelando la matrícula), que niños sigan estudios escolares, afectando el derecho a la educación de estos últimos.

Al respecto, el artículo $28^{\circ}$ de la Convención sobre los Derechos del Niño, señala expresamente que: "Los Estados partes reconocen el derecho del niño a la educación y, a fin de que se pueda ejercer progresivamente y en condiciones de igualdad de oportunidades ese derecho, deberán en particular: a) Implantar la enseñanza primaria obligatoria y gratuita para todos [...], d) hacer que todos los niños dispongan de información y orientación en cuestiones educacionales y profesionales y tengan acceso a ellas, e) Adoptar medidas para fomentar la asistencia regular a las escuelas y reducir las tasas de deserción escolar".

Es así que el Tribunal, en la Sentencia Nº52-2004-AA/TC, la cual se trata de la acción de amparo que interpone doña Martha Elena Cueva Morales a favor de su menor hijo J.J.C.C. de siete años de edad, y la dirige contra Richard Astiquilca Mayhuire, Director de la Escuela Primaria de Menores N 5006, alegando que se le ha lesionado el derecho a la educación del favorecido, pues el demandado se ha negado a ratificar su matrícula en el referido Centro Educativo.

Previamente el Tribunal precisa que, resulta indiscutible que, si efectivamente el demandado hubiera impedido que el beneficiario se matriculara para seguir en el siguiente año escolar, se habría afectado el derecho a la educación del 
favorecido; siendo que no solo se trata de un deber de los padres para con sus hijos, sino también un derecho (el de la educación) que cabe oponer y exigir al Estado.

"Si la Constitución ha establecido que los padres tienen el deber de brindar educación a sus hijos, respecto al Estado ha declarado que este está en la obligación de proteger especialmente al niño y al adolescente. Naturalmente esta protección especial implica primeramente la obligación de permitirle ingresar a un centro educativo, así como que se adopten todas las medidas necesarias y oportunas destinadas a impedir que nadie se vea impedido de recibir educación adecuada por razón de su situación económica o de limitaciones mentales o físicas". Evidentemente, se incumple ese deber especial, por ejemplo, cuando el Estado, a través de sus órganos y funcionarios competentes, niega a un menor la posibilidad de continuar sus estudios, sin existir motivos razonables para ello.

En esta resolución, el Tribunal resalta el valor instrumental del artículo 3.1. de la Convención sobre los Derechos del Niño, a tenor del cual: "En todas las medidas concernientes a los niños que tomen las instituciones públicas y privadas de bienestar social, los tribunales, las autoridades administrativas o los órganos legislativos, una consideración primordial a que se atendrá será: el interés superior del niño".

\section{c) El interés superior del niño y el derecho a la libertad personal}

Se trata de un proceso de hábeas corpus interpuesto por don Donato Villacorta Sánchez a favor de su hijo Alex Evert Villacorta Oviedo, contra la titular del Segundo Juzgado de Familia de la Corte Superior de Justicia del Cusco, solicitando se disponga la inmediata libertad del beneficiario, quien se encuentra internado en un centro de rehabilitación de menores, desde el 04 de noviembre del año 2002 hasta la fecha de la interposición de la demanda, sin que se haya resuelto su situación jurídica.

El demandante refiere que su hijo fue injustamente comprendido en la investigación por el presunto delito contra la libertad sexual en agravio de una menor, en la cual, la demandada dispuso su internamiento en un centro de menores, lugar donde se encuentra detenido más de ocho meses, lo que contraviene el artículo $221^{\circ}$ del Código de los Niños y Adolescentes.

El Tribunal señala que, en general, el hábeas corpus es un mecanismo procesal específico de tutela de la libertad y seguridad personal y derechos conexos; 
siendo que, en el presente caso, se está ante un hábeas corpus traslativo, cuando se mantenga indebidamente la privación de la libertad de una persona, o se demore la determinación jurisdiccional que resuelva la situación personal del detenido. En tal sentido, son materia de análisis los plazos en los que se ha llevado a cabo la investigación a que se encontraba sujeto el beneficiario. De dicho análisis se desprende, que han transcurrido más de siete meses desde el internamiento del menor sin que hubiera concluido el procedimiento; en consecuencia, el plazo máximo de cincuenta días se encontraba vencido en exceso. Por tales consideraciones e invocando al artículo IX del Título Preliminar del Código de los Niños y Adolescentes, el Tribunal declaró fundada la demanda, es decir, recoge el artículo $37^{\circ}$ de la Convención sobre los Derechos del Niño, el cual reconoce el derecho del niño a ser protegido contra la privación ilegal o arbitraria a su libertad, pudiendo impugnar la legalidad de tal medida ante un tribunal u otra autoridad competente, independiente e imparcial. Así se señala que los Estados partes velarán porque: b) ningún niño sea privado de su libertad ilegal o arbitrariamente. La detención, el encarcelamiento o la prisión de un niño se llevarán a cabo de conformidad con la ley y se utilizará tan solo como medida de último recurso y durante un periodo más breve que proceda.

\section{DETERMINACIÓN DEL INTERÉS SUPERIOR DEL NIÑO PARA EL TRIBUNAL CONSTITUCIONAL}

“Finalmente luego de la revisión de las sentencias del Tribunal Constitucional, aunque puede ser poco arriesgado hacerlo, permite realizar el planteamiento de indicaciones concretas que se detallan a continuación" ${ }^{8}$ :

a) El interés superior del niño, exigirá proveer en primer lugar a sus problemas inmediatos y más graves (salud, educación, libertad personal, etc.)

b) En la concreción de ese interés y con la pretensión de garantizarle sus derechos fundamentales, habrá de conjugarse, por principio e inicialmente, las necesidades presentes con las futuras que quepa razonablemente proveer.

c) Es preciso incentivar la autonomía personal y funcional en la definición de su interés, por ello la alternativa que se elija, debe ser aquella que haga efectivo el ejercicio de sus derechos fundamentales.

d) La objetividad que se demanda al momento de apreciar el interés superior del niño, es un requisito consustancial para determinar lo más favorable, lo mejor al resolver el caso (el criterio de objetividad está referido a tomar conocimiento de la opinión del niño, a conocer qué trato le brindan las 
personas de su entorno, familiares, conocidos, a la empatía con estas personas para con él, a las condiciones económicas y sociales que le rodean, al grado de desarrollo educativo alcanzado, etc.) entre otros aspectos.

\section{EL INTERÉS SUPERIOR DEL NIÑO EN EL PROCESO CIVIL, TUTELAR Y DE FAMILIA}

Ahora bien, luego de haber analizado, el principio del interés superior del niño desde una perspectiva formal, amplia, legislativa-jurisdiccional y de implicancia general, debe abordarse la intervención de tal principio en el contexto del proceso civil peruano y su comprensión por parte de los operadores jurisdiccionales.

Sin lugar a dudas, el proceso civil peruano, es eminentemente formal, debiéndose cumplir con todos los requisitos de procedibilidad y admisibilidad que exige nuestra norma procesal civil, teniendo como sustento para el planteamiento de diversas acciones, el sustento normativo, que para tal efecto, otorga, tanto el Código Civil, como el Código de los Niños y Adolescentes.

Es allí que todo proceso civil, debe en primer término cumplir con garantizar el derecho de las partes intervinientes, y en el marco de la observancia de un debido proceso.

Sin embargo, y contando para ello con la experiencia profesional, así como la experiencia procesal de la comunidad jurídica, se puede advertir que en los procesos civiles y en los cuales se encuentran inmersos menores de edad, efectivamente se garantiza el irrestricto derecho de defensa de los involucrados (partes procesales), se garantiza el cumplimiento irrestricto del debido proceso y además se cumple a cabalidad con los requerimientos tanto procesales como sustantivos; sin embargo, se deja de lado al verdadero autor del proceso, como es el menor de edad, siendo en muchos casos, el verdadero perjudicado; es decir, en tal situación, no se toma en cuenta el principio del interés superior del niño; o se tiene una distorsión del significado de dicho principio.

Ello ocurre muy a menudo en la etapa de conciliación, en donde el juez en ejercicio de sus atribuciones, trata de solucionar el conflicto suscitado (tenencia de un menor, alimentos, régimen de visitas, etc.), proponiendo una fórmula de conciliación que resuelva en definitiva la controversia, sin tener una visión clara y amplia de los hechos controversiales, escuchando únicamente a las partes involucradas, sin siquiera escuchar la opinión del menor. En otras 
situaciones, el juez adelanta opinión, al proponer una fórmula de conciliación, la cual no resulta beneficiosa para el menor; $y$, finalmente en otras, se advierte con suma claridad que, la fórmula de solución propuesta por el juez, no armoniza con lo resuelto en definitiva por el magistrado.

En un análisis exhaustivo del actuar del juez en el proceso civil de familia, se puede apreciar que, su actuación en tal etapa resulta importantísima y a la vez complicada, por cuanto debe valorar anticipadamente el caso concreto.

Sin embargo, resulta necesario una comprensión cabal a cargo del juez, del significado integral del principio del interés superior del niño; asimismo, resulta de suma importancia, comprender lo trascendente de su actuación en tal etapa procesal, pues el principio del interés superior del niño, no es un mero principio enunciativo que debe ser invocado por doquier, o cuando haya dificultad de escoger un presupuesto normativo que encuadre en tal o cual situación, ni tampoco debe utilizarse de manera equívoca. Por el contrario, dicho principio debe ser conocido y comprendido a cabalidad, en su verdadera dimensión y de contenido supralegal, debe además comprenderse con ello, las garantías y resultados que emanan de un debido proceso judicial, el respeto de todos los principios que inspiran la función jurisdiccional, teniendo siempre como visión orientadora al menor de edad, pues recién allí, se podrá hablar de un debido proceso con el respeto de todas las garantías y principios que consagra nuestra Constitución Política.

"Al Poder Judicial y en su caso al Tribunal Constitucional, normalmente le cabe un papel subsidiario: le corresponde actuar cuando los demás poderes incumplan con las obligaciones a su cargo, sea por su propia acción, por no poder evitar que otros particulares afecten el bien que constituye el objeto del derecho, o por incumplir con las acciones positivas debidas. Pero en el caso concreto de los niños y adolescentes es imprescindible que se ponga a trabajar activamente desde ahora mismo. No se necesita un juez "boca de ley" sino un activista judicial, que dé sentido a todos y cada uno de los derechos fundamentales, entendiendo el significado de la Constitución" ${ }^{\prime 9}$.

\section{CONCLUSIONES FINALES}

a) El derecho procesal de familia se ha consolidado en la última década como ente protector de la familia, niño, niña y adolescente.

b) El derecho procesal es la base de la consolidación de esta rama del derecho, pero no debe perderse de vista que esta última cuenta con características y elementos propios y cuyo sustento son valores y principios éticos. 
Marco Antonio Celis Vásquez - Hacia la consolidación del Derecho Procesal de Familia

c) El modelo de interpretación jurídica dinámica es el modelo a seguir por un Juez Especializado de Familia, si desea administrar justicia en materia familiar tutelando el derecho de niños, niñas y adolescentes.

d) El principio del interés superior del niño debe ser conocido y comprendido a cabalidad, en su verdadera dimensión y de contenido supralegal, y al momento de su apreciación debe primar la objetividad, como un requisito consustancial para determinar lo más favorable para un menor al resolver un caso determinado.

1 Palacio, Lino y Roland Arazi. Derecho procesal de familia. T. I. Buenos Aires: Rubinzal Culzoni Editores, 2002, pp. 16-17.

2 Morillo, Augusto. La influencia de los procesos de familia sobre la litigación civil, citado por Palacio, Lino y Roland Arazi. Ob. Cit., p. 17.

3 Taruffo, Michele. La motivación de la sentencia civil. Mexico: Editorial del Tribunal del Poder Judicial de la Federación, 2006, p. 124.

4 Kemelmajer De Carlucci, Aida. Principios procesales y tribunales de familia, citado por Palacio, Lino y Roland Arazi. Ob. Cit., p. 32.

5 Plácido Vilcachagua, Alex. Las causales de divorcio y separación de cuerpos en la jurisprudencia civil. Lima: Gaceta Jurídica, 2008, pp. 213-216.

6 Montoya Chávez, Víctor Hugo. Derechos fundamentales de los niños y adolescentes. El interés superior del niño y adolescente y la situación de abandono en el artículo $4^{\circ}$ de la Constitución. Lima: Grijley, 2007, pp. 50-53.

7 Sentencia emitida por el Tribunal Constitucional recaída en el Expediente $N^{\circ}$ 2165-2005-HC/TC, de fecha 14 de octubre de 2002.

8 Plácido Vilcachagua, Alex. Temas de derecho de familia y del niño. Elaborado en el XIII Convocatoria de cursos a distancia para magistrados - Academia de la Magistratura, 2007, p. 160.

9 Montoya Chávez, Víctor Hugo. Ob. Cit., pp. 288-289. 